\title{
ANÁLISIS HIDROBIOLÓGICO Y SANITARIO DE LA QUEBRADA SANTA MÓNICA, CONCORDIA ANTIOQUIA-COLOMBIA
}

\author{
Noris Emilse Quintero', Juan Pablo Serna², Fabio de Jesús Vélez ${ }^{3}$, Néstor Jaime Aguirre ${ }^{4}$ \\ ${ }^{1}$ Ingeniera Sanitaria. Escuela Ambiental, Universidad de Antioquia. Grupo GeoLimna noris.quintero@udea.edu.co. \\ 2 Doctor en Ingeniería Ambiental. Profesor Escuela Ambiental, Universidad de Antioquia. Grupo GeoLimna. jpa- \\ blo.sera@udea.edu.co. \\ 3Doctor en Geografía. Profesor Escuela Ambiental, Universidad de Antioquia. Grupo GeoLimna. fabio.ve- \\ lez@udea.edu.co. \\ ${ }^{4}$ Doctor en Limnología. Profesor Escuela Ambiental, Universidad de Antioquia. Grupo GeoLimna nestor.agui- \\ rre@udea.edu.co.
}

\section{RESUMEN}

La Quebrada Santa Mónica es la fuente con el mayor aporte del embalse la Nitrera. Este abastece de agua potable al municipio de Concordia. Con el propósito de analizar la calidad del agua de la quebrada y determinar si cumple con los criterios de calidad del RAS, se realizó la caracterización fisicoquímica y biológica del agua empleando los macroinvertebrados acuáticos como bioindicadores. En tres muestreos en el año 2017, en tres estaciones de la quebrada, se tomaron muestras fisicoquímicas e hidrobiológicas. Los resultados fisicoquímicos presentaron una variación espaciotemporal leve; se pudo determinar que el mes de noviembre presentó el valor más alto de abundancia y riqueza de macroinvertebrados, siendo Phylloicus la taxa más abundante. El índice BWWP/Col estuvo entre 52 y 119. La Quebrada Santa Mónica presenta buena calidad de agua y la mayoría de los parámetros fisicoquímicos cumplen con la normatividad para ser fuente de abastecimiento de agua potable.

Palabras clave: Macroinvertebrados acuáticos; Índices biológicos; Análisis fisicoquímicos; Calidad del agua

Recibido: 26 de octubre de 2020. Aceptado: 09 de abril de 2021

Received: October 26, 2020. Accepted: April 09, 2021

DOI: https://doi.org/10.33571/rpolitec.v17n33a1

\section{HYDROBIOLOGICAL AND SANITARY ANALYSIS OF THE SANTA MONICA CREEK, CONCORDIA, AN- TIOQUIA-COLOMBIA}

\begin{abstract}
The Santa Monica creek is the main source of the Nitrera reservoir, which supplies the drinking water to the municipality of Concordia. To determine the quality of the water in the Creek, and if it accomplish the standards, a physical-chemical and biological characterization was carried out using aquatic macroinvertebrates as bioindicators. Physical-chemical and hydro-biological samples were taken at three stations in the creek during three campaigns in 2017. The physicochemical results presented a slight spatial-temporal variation, and we determined that November presented the highest abundance and richness values of macroinvertebrates, being Phylloicus the predominant taxa. The BWWP/Col index values were between 52 and 119. Santa Mónica Creek has a good water quality, and most of the physical-chemical parameters accomplish the regulations to be a source for drinkable water supply.
\end{abstract}

Keywords: Aquatic macroinvertebrates; Biological indices; Physical-chemical analysis; Water quality.

Cómo citar este artículo: N.E. Quintero, J.P. Serna, F.J. Vélez, N.J. Aguirre. "Análisis hidrobiológico y sanitario de la quebrada Santa Mónica, Concordia Antioquia-Colombia”, Revista Politécnica, vol.17, no.33 pp.9-21, 2021. DOI: https://doi.org/10.33571/rpolitec.v17n33a1 


\section{INTRODUCCIÓN}

El análisis de la información de calidad del agua derivada de variables fisicoquímicas, de los macroinvertebrados acuáticos y el cálculo de índices, permiten obtener un conocimiento sobre el estado actual del agua y sus posibles tendencias. Las variables fisicoquímicas permiten conocer la calidad del agua en el instante del muestreo, en tanto que los macroinvertebrados acuáticos permiten determinar las condiciones que prevalecen en el ambiente por más tiempo, siendo estos unos los organismos más empleados para evaluar el grado de saprobiedad presente en corrientes de agua [1], [2], estos organismos se pueden utilizar como bioindicadores de la calidad del agua, hecho reconocido también para evaluar las condiciones ambientales de los ecosistemas lóticos [3]-[6].

El empleo de comunidades acuáticas como bioindicadores de la calidad del agua está asociado a la aplicación de los índices de diversidad como son: el índice de diversidad Shannon-Weaver, 1949, dominancia de Simpson, 1977 y el equidad de Pielou, 1977; como también índices comparativos-cualitativos, como los índices de Jaccard y Sorensen, los cuales se emplean para evaluar la respuesta de los macroinvertebrados acuáticos a la calidad ambiental del agua, a partir de la comparación de comunidades entre diferentes sitios de muestreo [7]-[10]. Entre las ventajas de los bioindicadores están la facilidad en la identificación de los organismos, y un amplio conjunto de ríos evaluados en el departamento de Antioquia y el país [11].

Dado que la quebrada Santa Mónica es una fuente importante para el abastecimiento de agua del municipio de Concordia, y hasta el momento solo se tenían registros de aforos realizados en el año 2016 [12], fue necesario realizar estudios que permitan conocer la calidad del agua de dicha fuente; por lo que se planteó la siguiente pregunta de investigación: ¿Cómo es la calidad del recurso hídrico en la Quebrada Santa Mónica en el parque natural La Nitrera en Concordia, Antioquia?

Esta investigación planteó como hipótesis, que es posible determinar la calidad del recurso hídrico de la Quebrada Santa Mónica a través del análisis de variables fisicoquímicas e hidrobiológicas utilizando macroinvertebrados acuáticos como bioindicadores. El propósito de esta investigación fue realizar la caracterización hidrobiológica y sanitaria de la quebrada Santa Mónica, a través del análisis de la calidad del agua, para determinar si esta es una fuente apta para abastecimiento de agua para consumo humano según el RAS 2017[13]

\section{MATERIALES Y METODO}

Área de estudio: El embalse "Miguel Martínez Isaza" está ubicado en la reserva forestal La Nitrera en el municipio de Concordia Antioquia. La reserva forestal comprende un área total actual de 101,5 hectáreas de extensión, está a una altitud media de 2161 m.s.n.m.; la temperatura promedio es de $17^{\circ} \mathrm{C}$ y la precipitación media es de $2000 \mathrm{~mm} / \mathrm{año}$. La estructura de contención se encuentra a unos $4,5 \mathrm{~km}$ al suroeste de la cabecera urbana en donde convergen las aguas de las quebradas Santa Mónica, San Luis y La Nitrera que le da el nombre (Fig. 1.). Dicho embalse es la única fuente del acueducto municipal de Concordia, Antioquia [14]. La quebrada Santa Mónica es la mayor aportante de caudal, toda vez que cuenta con un área de drenaje de $0,66 \mathrm{~km} 2$, de los $0,96 \mathrm{~km} 2$ en total que tiene la microcuenca que surte al embalse; esto corresponde al $68,7 \%$ del área total. Dicha quebrada tiene además una longitud de $1230 \mathrm{~m}$. El embalse cuenta con una capacidad de almacenamiento de aproximadamente $76.850 \mathrm{~m} 3$ y la concesión es de 32,5 L/s [12]. 


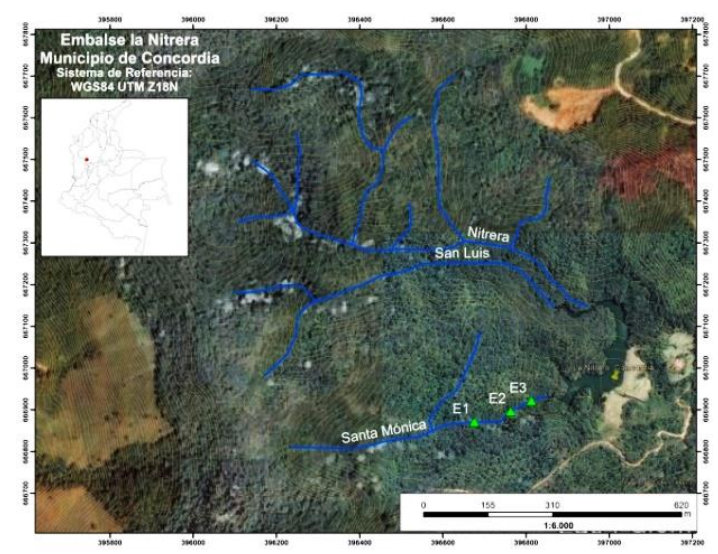

Fig. 1. Cauce aproximado de la quebrada Santa Mónica y ubicación geográfica de sitios de muestreo (Fuentes: DEM Alos Palsar, y Google Earth, editada por Fabio Vélez).

Se realizaron tres campañas de muestreo: la primera el 14 de febrero de 2017; la segunda el 17 de agosto de 2017, y la tercera el 28 de noviembre de 2017. La primera se utilizó a la vez como una visita de inspección con el propósito de elegir los tres puntos de muestreo; allí se tomaron las primeras muestras de macroinvertebrados y se midieron algunas variables fisicoquímicas.

Las estaciones de muestreo denominadas E1, E2 y E3 se localizaron como lo muestra la Figura 1, siendo E1 la más lejana aguas arriba, luego E2 a 15,60 m aguas abajo de E1, y E3 situada cerca a la desembocadura al embalse la Nitrera, a 25,40 m aguas abajo de E2. La Tabla 1 presenta una descripción de cada una de las estaciones, necesaria para el análisis.

Tabla 1. Descripción estaciones de muestreo de la Quebrada Santa Mónica.

\begin{tabular}{|c|c|c|c|c|}
\hline \multirow{2}{*}{ Estación } & \multirow{2}{*}{ Descripción } & \multicolumn{2}{|c|}{ Coordenadas } & \multirow{2}{*}{$\begin{array}{c}\text { Altura } \\
\text { m.s.n.m }\end{array}$} \\
\hline & & Latitud & Longitud & \\
\hline E1 & Cascada, lecho sobre sustrato rocoso & $6^{\circ} 1 ' 57,6^{\prime \prime} \mathrm{N}$ & $75^{\circ} 56^{\prime} 12,8^{\prime \prime} \mathrm{W}$ & 2.180 \\
\hline E2 & Curva izquierda, lecho sobre sustrato grava & $6^{\circ} 1^{\prime} 57^{\prime} \mathrm{N}$ & $75^{\circ} 56^{\prime} 1,6^{\prime \prime} W$ & 2.160 \\
\hline E3 & Cerca al embalse la Nitrera, sustrato arenoso & $6^{\circ} 1 ' 57,8$ " N & $75^{\circ} 55^{\prime} 56,5^{\prime \prime} \mathrm{W}$ & 2.160 \\
\hline
\end{tabular}

- Determinación del perfil del cauce

Mediante una topografía elemental con equipos tales como mira topográfica, nivel Locke, lienza, brújula, y GPS, se pudieron determinar las distancias, alturas y ángulos, con los cuales se calcularon los desniveles entre estaciones y los de los taludes aferentes, para posteriormente, mediante Excel, obtener el perfil topográfico.

- Determinación de variables hidráulicas

La medición de caudal se realizó por el método integral del polígono (área-velocidad); este método consiste en medir, en cada estación, el área tranversal y la velocidad del agua en cada subsección definida. Para el caso, y dada la poca profundidad y el relativo bajo caudal, se utilizó una esfera plástica y un cronómetro para medir la velocidad promedio en cada sección, y para el área, se midió el ancho superficial, este se dividió en sendas porciones iguales a las cuales se les midió la profundidad de la columna de agua; finalmente se integran todos los segmento y se obtiene el área de cada estación; el cálculo del caudal se obtiene a partir de la ecuación

$\mathrm{Q}=v^{*} \Sigma \alpha_{1}$

[15] donde $Q$ es el caudal, v la velocidad promedio y ai el área de cada subsección.

- Determinación de las variables fisicoquímicas

En cada sitio de muestreo se registraron variables fisicoquímicas in situ como temperatura, conductividad, oxígeno disuelto y $\mathrm{pH}$. Además, se tomaron muestras de agua para ser analizadas usando Kits Visocolor 
para determinar alcalinidad, dureza, nitratos y nitritos; las muestras para turbiedad y DQO fueron analizadas en el laboratorio Hidrobiología Sanitaria de la Universidad de Antioquia. En la Tabla 2 se presenta la lista de variables determinadas y su respectivo método.

Tabla 2. Variables fisicoquímicas y bióticas analizadas en la Quebrada Santa Mónica (Concordia- Antioquia).

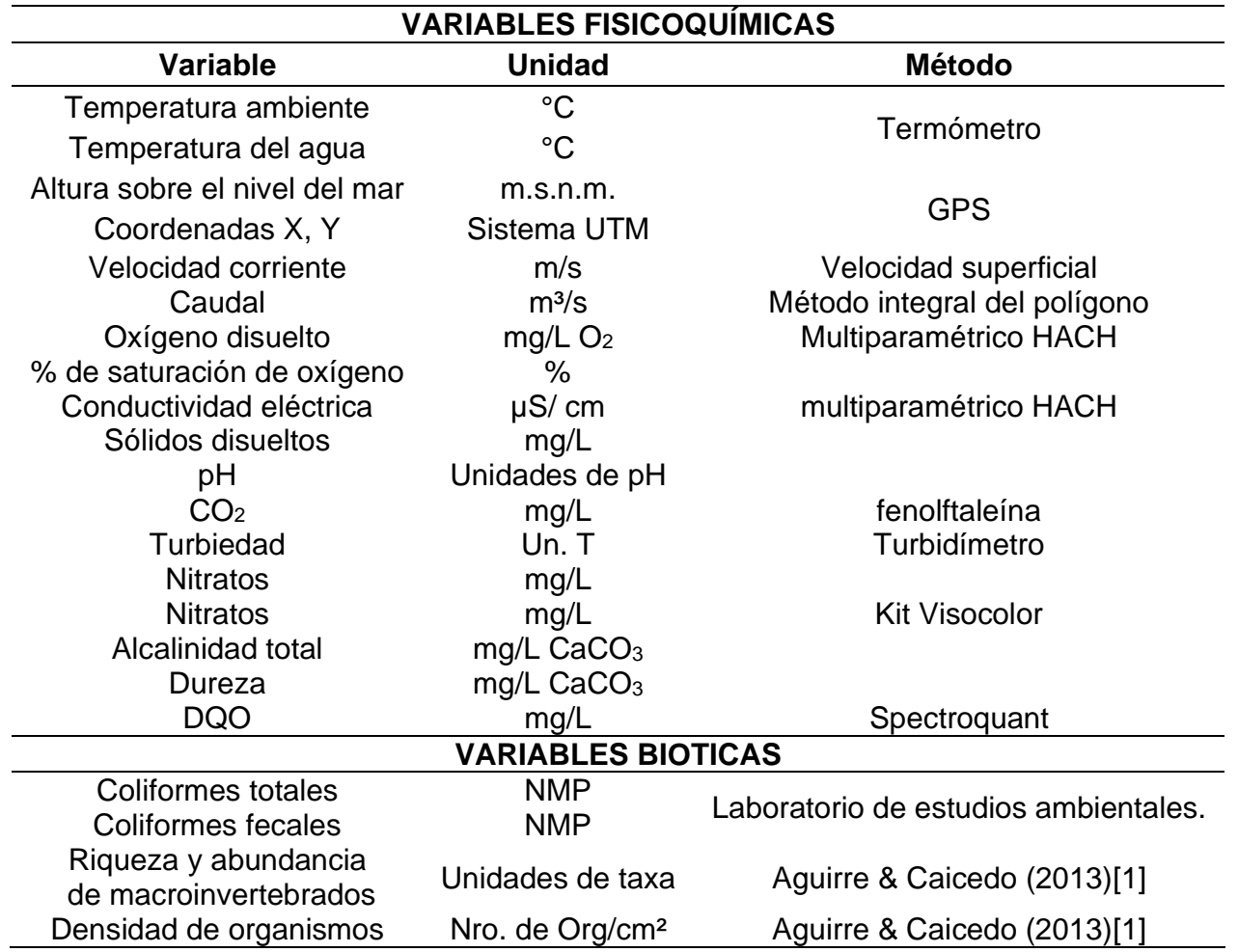

- Determinación de variables bióticas

En cada estación se recolectaron macroinvertebrados acuáticos por medio de la red triangular, para muestras cualitativas, y la red Surber, para muestras cuantitativas. Por cada muestra obtenida se tomó la respectiva réplica. Las muestras fueron conservadas en alcohol y separadas para su posterior análisis en el laboratorio de Hidrobiología Sanitaria, usando un estereomicroscopio Karl Zeiss y claves de determinación taxonómicas [1], [6], [16]-[21] [22]. Para el estudio microbiológico, las muestras se analizaron en el laboratorio de estudios ambientales de la Universidad de Antioquia.

Con las muestras de macroinvertebrados acuáticos debidamente identificados y contabilizados, se determinaron los respectivos índices. Para cada estación se estimó el índice \% EPT [23], [24], y el índice BMWP_al igual que la riqueza, la abundancia y la densidad de individuos. Para estimar la diversidad de macroinvertebrados se emplearon los índices de dominancia de Simpson, diversidad de Shannon-Weaver y equidad de Pielou. También se analizó la similaridad entre las diferentes estaciones utilizando índice de similaridad de Jaccard (1908), Sörensen (1948) y el coeficiente de similitud [1]

\section{ANALISIS DE RESULTADOS}

Variables meteorológicas e hidráulicas

Mediante los registros históricos de precipitación de la estación más cercana a la cuenca de la quebrada Santa Mónica (Latitud $06^{\circ} 02^{\prime}$ N, Longitud $75^{\circ} 55$ W), desde el año 1970 al 2017 (Fig. 2.), se pudo observar que los máximos se presentan en el mes de abril, mientras que los mínimos ocurren entre los meses de enero y febrero; también se evidencia que históricamente el mes de noviembre presenta valores relativamente altos de precipitación. En los meses de muestreo se presentaron las siguientes situaciones: en agosto el máximo de precipitación mensual fue $28 \mathrm{~mm}$, y en noviembre se presentó un máximo de $80 \mathrm{~mm}$, lo que marca una 
situación contrastante para los tiempos de medición en campo [25]; esto puede tener una incidencia fuerte en la variación del caudal y en la disponibilidad de agua para el abastecimiento del municipio de Concordia.

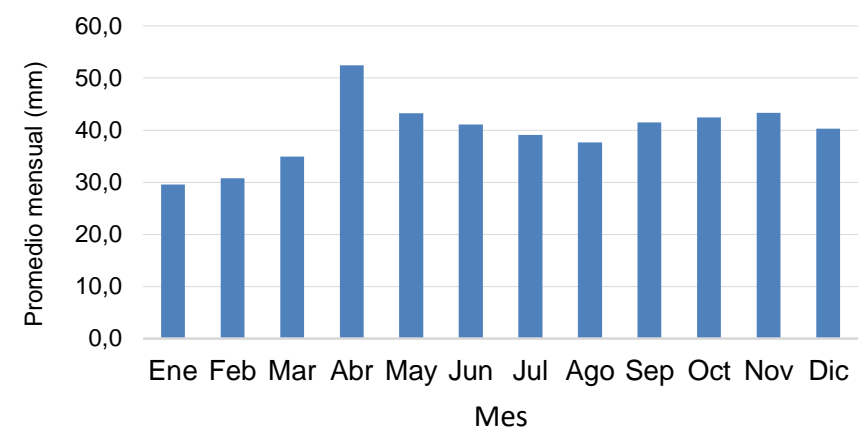

Fig. 2. Histograma de precipitaciones corriente Quebrada Magallo Municipio de Concordia (Fuente: Elaboración propia).

La quebrada Santa Mónica presentó una leve variación en la velocidad media de la corriente, para el mes de febrero: la velocidad en E3 fue mayor que en E2, y esta a su vez mayor que en E1; mientras que en el mes de agosto se comportó de manera inversa (Fig. 3.), con un rango entre 0,06 a 0,51 m/s. Esto pudo deberse a la configuración del canal natural y las características en cada sección. La velocidad más alta se dio en el mes de noviembre para la E3.

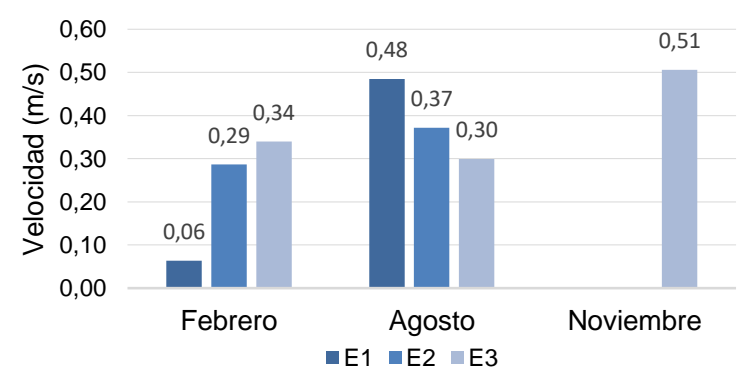

Fig. 3. Variación espaciotemporal del área mojada de la Quebrada Santa Mónica. (Fuente: Elaboración propia).

El caudal máximo se presentó igualmente en el mes de noviembre en E3 (Fig. 4.), lo cual pudo ocurrir por una mayor precipitación promedio mensual para ese período [25].

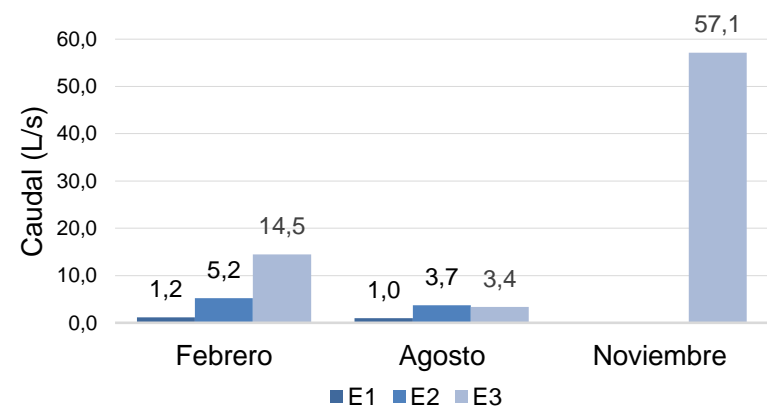

Fig. 4. Distribución espaciotemporal del Caudal de la Quebrada Santa Mónica.

(Fuente: Elaboración propia).

También se puede apreciar como las diferencias de caudal en los distintos muestreos variaron considerablemente, en un rango entre 1,0 y 57,15 L/s. El mes de febrero aportó mayores diferencias entre estaciones, mientras que en agosto se presentaron los caudales más bajos, lo que puede influir en la disponibilidad de agua del embalse para proveer al municipio, si se parte de la base de una demanda de agua per capita e 120 Litros/Habitante-día y una población urbana aproximada de 7.000 habitantes [12]. Estas situaciones de sequía, estacionales o eventuales, demuestran la importancia del embalse para el municipio. 


\section{Variables fisicoquímicas}

Para la primera campaña de muestreo sólo se contó con los datos de temperatura, oxígeno disuelto, conductividad y solidos disueltos. Se puede apreciar que, a excepción de la conductividad eléctrica, los sólidos disueltos, $\mathrm{CO}_{2}$, turbiedad y DQO, las demás variables no presentaron variaciones considerables, ni espacial ni temporalmente (Tabla 3). En cuanto a los datos estadísticos, los datos de varianza y desviación estándar son relativamente bajos para la mayoría de las variables. Las medidas que presentaron mayor varianza fueron el $\mathrm{CO}_{2}$ y la $\mathrm{DQO}$ con $\sigma^{2}=159,62$ y 538,67 respectivamente; estos valores tan altos pudieron darse debido a aportes de sustancias de interés sanitario, por el aumento en las lluvias (Tabla 4).

Tabla 3. Variables fisicoquímicas determinadas en la Quebrada Santa Mónica.

\begin{tabular}{|c|c|c|c|c|c|c|c|c|c|c|}
\hline \multirow{3}{*}{ Variables Físico Químicas } & \multicolumn{9}{|c|}{ PERIODOS DE MUESTREO Y ESTACIONES } & \multirow{3}{*}{$\begin{array}{c}\text { RAS } 2017 \\
\text { Máx. Admisible }\end{array}$} \\
\hline & \multicolumn{3}{|c|}{ Febrero } & \multicolumn{3}{|c|}{ Agosto } & \multicolumn{3}{|c|}{ Noviembre } & \\
\hline & E1 & E2 & E3 & E1 & E2 & E3 & E1 & E2 & E3 & \\
\hline Temperatura del agua ${ }^{\circ} \mathrm{C}$ & - & - & - & 18,15 & 18,1 & 18,3 & 16,7 & 17,6 & 17,4 & No exige \\
\hline Temperatura ambiente ${ }^{\circ} \mathrm{C}$ & 15,4 & 16,2 & - & 17,9 & 17,5 & 18 & 17 & 16,9 & 17 & No exige \\
\hline Oxígeno disuelto mg/L & 5,7 & 5,8 & 5,3 & 6,5 & 6,5 & 7,3 & 7,3 & 7,3 & 7,1 & 4 \\
\hline \% saturación de oxígeno & 56,8 & 58,4 & 49,9 & 68,3 & 68,3 & 75,9 & 74,0 & 75,5 & 73,1 & No exige \\
\hline Conductividad eléctrica $\mu \mathrm{s} / \mathrm{cm}$ & 23,3 & 44,0 & 37,8 & 41,3 & 40,8 & 40,7 & 34,4 & 59,9 & 33,2 & 1000 \\
\hline Sólidos disueltos mg/L & 19,1 & 36,1 & 31,0 & 33,9 & 33,5 & 33,4 & 28,2 & 49,2 & 27,2 & No exige \\
\hline $\mathrm{pH}$ & - & - & - & 7,1 & 7,2 & 7,4 & 8,2 & 8,9 & 8,1 & $6,5-9$ \\
\hline $\mathrm{CO}_{2} \mathrm{mg} / \mathrm{L}$ & - & - & - & 6,6 & 5,5 & 4,4 & 30,8 & 28,6 & 25,6 & No exige \\
\hline Turbiedad_UNT & - & - & - & 3,5 & 5,6 & 3,6 & 12,3 & 11,7 & 5,5 & 2 \\
\hline Nitratos $\mathrm{mg} / \mathrm{L}$ de $\mathrm{CaCO}_{3}$ & - & - & - & 1,0 & 0 & 1,2 & - & - & - & 10 \\
\hline Nitritos $\mathrm{mg} / \mathrm{L}$ de $\mathrm{CaCO}_{3}$ & - & - & - & 0,03 & 0,03 & 0,03 & - & - & - & 0,1 \\
\hline Alcalinidad total mg/L & 12,2 & 12,2 & 12,2 & 12,2 & 12,2 & 12,2 & 12,2 & 12,2 & 12,2 & 200 \\
\hline Dureza mg/L de $\mathrm{CaCO}_{3}$ & - & - & - & 15,0 & 15,0 & 15,0 & 18,0 & 18,0 & 18,0 & 300 \\
\hline $\mathrm{DQO} \mathrm{mg} / \mathrm{L}$ & - & - & - & 10,0 & 20,0 & 10,0 & 60,0 & 28,0 & 60,0 & 6 \\
\hline
\end{tabular}

Tabla 4. Valores estadísticos de las variables fisicoquímicas.

\begin{tabular}{cccccccc}
\hline & \multicolumn{6}{c}{ Valores estadísticos } \\
\cline { 2 - 7 } Variables Fisicoquímicas & $\mathbf{N}$ & $\mathbf{X}$ & $\boldsymbol{\sigma}^{2}$ & $\boldsymbol{\Sigma}$ & Mín. & Máx. \\
\cline { 2 - 7 } & 6 & 17,71 & 0,36 & 0,6 & 16,7 & 18,3 \\
Temperatura del agua $\left({ }^{\circ} \mathrm{C}\right)$ & 9 & 16,99 & 0,75 & 0,87 & 15,4 & 18 \\
Temperatura ambiente ${ }^{\circ} \mathrm{C}$ & 9 & 6,53 & 0,61 & 0,78 & 5,3 & 7,3 \\
Oxígeno disuelto (mg/L) & 9 & & & & & & \\
Saturación de oxígeno (\%) & 9 & 66,69 & 88,82 & 9,42 & 49,9 & 75,9 \\
& & & & & & \\
Conductividad eléctrica $(\mu \mathrm{s} / \mathrm{cm})$ & 9 & 39,49 & 96,73 & 9,83 & 23,3 & 59,9 \\
Sólidos disueltos (mg/L) & 6 & 32,4 & 65,49 & 8,09 & 19,1 & 49,2
\end{tabular}




\begin{tabular}{ccccccc}
$\mathrm{pH}$ & 6 & 7,82 & 0,49 & 0,7 & 7,1 & 8,9 \\
$\mathrm{CO}_{2}(\mathrm{mg} / \mathrm{L})$ & 6 & 16,92 & 159,62 & 12,63 & 4,4 & 30,8 \\
& 3 & 7,03 & 15,64 & 3,95 & 3,5 & 12,3 \\
\hline Turbiedad (NFU) & 3 & & & & & \\
\hline
\end{tabular}

La temperatura tanto ambiental como la del agua no presentó cambios significativos ni en el espacio ni en el tiempo, estando en un rango entre 15,4 y $18,3^{\circ} \mathrm{C}$. Del mismo modo la concentración de nutrientes no presentó variaciones espaciales en la campaña del mes de agosto, estando la concentración de nitritos estable para las tres estaciones, $0,03 \mathrm{mg} / \mathrm{L}$, mientras que la de nitratos estuvo entre 1,0 y 1,2 mg/L, lo que implica un bajo contenido de nutrientes en el agua, dado el hecho de que hay poca intervención antrópica en el lugar. Tanto las concentraciones de nitritos como de nitratos para los diferentes periodos tuvieron valores aceptables [13].

Los valores obtenidos de dureza en agosto y noviembre fueron de 15 y de $18 \mathrm{mg} / \mathrm{L}$ respectivamente, sin variación entre estaciones en cada uno de los muestreos; dichas concentraciones están por debajo del valor máximo admisible [13]. En cuanto a la alcalinidad se obtuvo un valor constante de $12,2 \mathrm{mg} / \mathrm{L}$ tanto en febrero como en agosto, en las tres estaciones. En cuanto al pH se presentaron valores entre 7,16 y 8,14 unidades, que están dentro del rango admisible según el RAS 2017, que está entre 6,5 y 9,0. Valores por fuera de este rango pueden ocasionar alteraciones en la fauna y la flora [26].

La DQO mostró una pequeña variación espacial en el mes de agosto con $10 \mathrm{mg} / \mathrm{L}$ en E1 y E3 y de $20 \mathrm{mg} / \mathrm{L}$ en E2. Esto significa una calidad de agua con bajo contenido de materia orgánica. En el mes de noviembre, E1 y E3 presentaron una concentración de $60 \mathrm{mg} / \mathrm{L}$ ambas, mientras que en E2 fue de $28 \mathrm{mg} / \mathrm{L}$. Estos valores son mayores, y según el RAS 2017, el agua con estas características, necesita ser tratada para ser consumida [13].

La concentración de oxígeno en el cuerpo de agua presentó valores mínimos y máximos de 5,3 y 7,29 mg/L respectivamente, en los diferentes muestreos, aumentando en el tiempo, pero con pocas variaciones entre estaciones. En cuanto a la temperatura y la altitud del embalse sobre el nivel del mar, se puede decir que sus valores propician una baja cinética de degradación de la materia orgánica. El porcentaje de saturación de oxígeno por su parte se situó en el rango entre 49,1\% y 110,0\%, lo cual refleja un ecosistema en equilibrio.

En cuanto al $\mathrm{CO}_{2}$, se encontraron concentraciones altas, con valores en el intervalo entre 4,4 y $30,8 \mathrm{mg} / \mathrm{L}$, lo que puede representar problemas de corrosión en las estructuras del embalse. Los mayores valores observados en las tres estaciones, en el mes de noviembre, puede deberse a tres aspectos: la descomposición de materia orgánica presente en el agua, en especial hojas y troncos de árbol; a un mayor aporte de aguas lluvias, y a la respiración de la vida acuática, siendo el primer aspecto, el más conspicuo y representativo de las condiciones observadas.

Los valores obtenidos para la turbiedad del agua en cada estación y cada campaña están en un rango entre 3,36 y 12,9 UNT, lo cual la cataloga como de calidad regular, según el RAS 2007, que requiere de tratamiento de potabilización para cumplir con el parámetro de 2 UNT, aceptable para el consumo humano. El valor máximo admisible por el RAS 2017 para conductividad eléctrica es de $1000 \mu \mathrm{s} / \mathrm{cm}$; los valores obtenidos para dicho parámetro están en un rango de 23,3 a 43,97 $\mu \mathrm{s} / \mathrm{cm}$, muy por debajo del límite.

La medición de los sólidos disueltos se hizo indirectamente a partir de la conductividad eléctrica, obteniéndose valores en un rango entre 19,13 a 49,20 mg/L. Se puede notar que son valores relativamente bajos, y sin cambios significativos en el espacio y tiempo.

2.

\section{Variables bióticas}

3. 
Las variables bióticas que se midieron fueron, una de tipo microbiológico, y otra de macroinvertebrados acuáticos. El análisis microbiológico que se realizó solo en la estación E3 fue de coliformes fecales y totales, ya que esta estación es la más cercana al embalse; según los resultados del Laboratorio de Estudios Ambientales, se reportó la presencia de coliformes fecales y totales para las dos campañas de muestreo (Tabla 5). Este resultado puede estar asociado a la presencia de fauna natural, o de animales domésticos abandonados en la reserva, como es el caso de perros ferales. Según el RAS 2017, no puede haber presencia de este tipo de microorganismos en aguas destinadas para el consumo humano, por ser indicadores de presencia de patógenos, lo que hace necesario aplicar tratamientos convencionales y desinfección antes de ser consumida [13].

Tabla 5. Resultados de Coliformes fecales y totales en la Quebrada. Santa Mónica.

\begin{tabular}{ccc}
\hline FECHA & $\begin{array}{c}\text { COLIFORMES } \\
\text { FECALES } \\
\text { (NMP/100ml) }\end{array}$ & $\begin{array}{c}\text { COLIFORMES } \\
\text { TOTALES } \\
\text { (NMP/100ml) }\end{array}$ \\
\hline $\begin{array}{c}\text { Ago } \\
2017\end{array}$ & $8^{*} 10 \wedge 1$ & $193^{\star} 10 \wedge 1$ \\
Nov & $6 * 10 \wedge 1$ & $435^{\star} 10 \wedge 1$ \\
2017 & 6 & \\
\hline
\end{tabular}

Mediante la presencia de macroinvertebrados acuáticos se determinó el estado de calidad de la quebrada. En la Tabla 6 se presentan los taxa de las muestras cualitativas de las tres campañas, donde se encontraron 22 morfotipos de los cuales 18 se pudieron identificar hasta nivel de género, para un total de 40 individuos, distribuidos así: 27 en E1, 12 en E2 y 12 en E3. La estación en la que se halló más riqueza de morfotipos fue en la E1, con un total de 14, seguida de E2 y E3 con 10 respectivamente. Las familias que predominaron en las tres estaciones fueron Perlidae, Calamoceratidae, Veliidae, Chironomidae y Gomphidae. Las tres primeras son indicadoras de aguas limpias u oligosaprobias y la última de aguas oligo-mesosaprobias [16].

Tabla 6. Macroinvertebrados acuáticos encontrados en las diferentes estaciones de muestreo (muestreo cualitativo).

\begin{tabular}{|c|c|c|c|c|c|}
\hline \multirow{2}{*}{ ÓRDEN } & \multirow{2}{*}{ FAMILIA } & \multirow{2}{*}{ GÉNERO } & \multicolumn{3}{|c|}{ ESTACIONES } \\
\hline & & & E1 & E2 & E3 \\
\hline \multirow{3}{*}{ Coleóptera } & Ptilodactylidae & Anchytarsus & 2 & & \\
\hline & Lampyridae & Sin determinar & & 1 & 1 \\
\hline & Hydropsychidae & Sin determinar & 3 & & 1 \\
\hline \multirow{5}{*}{ Díptera } & Dixidae & Dixella & 1 & & \\
\hline & Chironomidae & Sin determinar & 1 & 1 & 1 \\
\hline & Simuliidae & Simulium & 5 & & \\
\hline & Tipulidae & Hexatoma & 1 & & 1 \\
\hline & Chironomidae & Tanypodinae (SubFlia) & & & 3 \\
\hline \multirow{2}{*}{ Ephemeróptera } & Baetidae & Baetodes & & 1 & \\
\hline & Baetidae & Baetis & & 1 & \\
\hline \multirow{4}{*}{ Hemíptera } & Veliidae & Rhagovelia & 1 & 2 & 1 \\
\hline & Gerridae & Eurygerris & 2 & 1 & \\
\hline & Naucoridae & Limnocoris & & 2 & \\
\hline & Gomphidae & Progomphus & & 1 & 1 \\
\hline \multirow[t]{2}{*}{ Odonata } & Polythoridae & Polythore & 1 & & \\
\hline & Calopterygidae & Hetaerina & 2 & & \\
\hline \multirow[t]{2}{*}{ Plecóptera } & Perlidae & Anacroneuria & 2 & 1 & 1 \\
\hline & Calamoceratidae & Phylloicus & 2 & 1 & 1 \\
\hline \multirow[t]{2}{*}{ Trichóptera } & Hydropsychidae & Sin determinar & 3 & & 1 \\
\hline & Glossosomatidae & Mortoniella & 1 & & \\
\hline \multicolumn{3}{|c|}{ \# TOTAL DE INDIVIDUOS } & 27 & 12 & 12 \\
\hline \multicolumn{3}{|c|}{ RIQUEZA DE MORFOTIPOS } & 14 & 10 & 10 \\
\hline
\end{tabular}

Sobre las muestras cuantitativas de las tres estaciones, en las tres campañas de muestreo, se pudieron identificar 26 morfotipos diferentes de un total de 166 individuos encontrados; sobresalen por su abundancia Phylloicus con 74 individuos, 44,6 \% del total, seguida de Anchytarsus sp. con 21, Anacroneuria sp. con 19 
individuos; todos son característicos de aguas oligotróficas [16]; la familia en menor proporción encontrada fue la Baetidae, con solo dos individuos.

Noviembre fue el mes donde se encontró la mayor abundancia y riqueza con un total de 100 individuos (Fig. 5.), 30 en E1, 57 en E2 y 13 en E3, respectivamente (Tabla 7). Los taxa más predominantes fueron Phylloicus sp. con 54 individuos correspondiente al $57,5 \%$ del total de individuos de dicha campaña, y Anchytarsus sp. con un total de 11 individuos representando el 11,7\%. En el mes de agosto se encontraron 38 individuos, 11 en E1, 8 en E2 y 19 en E3, los taxa que más predominaron fueron Anchytarsus sp. con 10 individuos, seguida de Anacroneuria sp. con 9 individuos. Finalmente, Phylloicus sp. con 8 individuos representando el 21,7, 19,6 y $17,4 \%$ respectivamente.

En el mes de febrero solo se hallaron 26 individuos, ya que solo se tomaron muestras en E2. Siendo Phylloicus sp. el taxa más representativo con un total de 12 individuos, y una participación del 46,15\% del total de individuos. El mes de mayor abundancia de organismos coincide con el aumento de caudal. En cuanto a la densidad de organismos, casi todas las estaciones mostraron densidades relativamente bajas; la única estación con densidad alta fue E2 en el mes de noviembre [3].

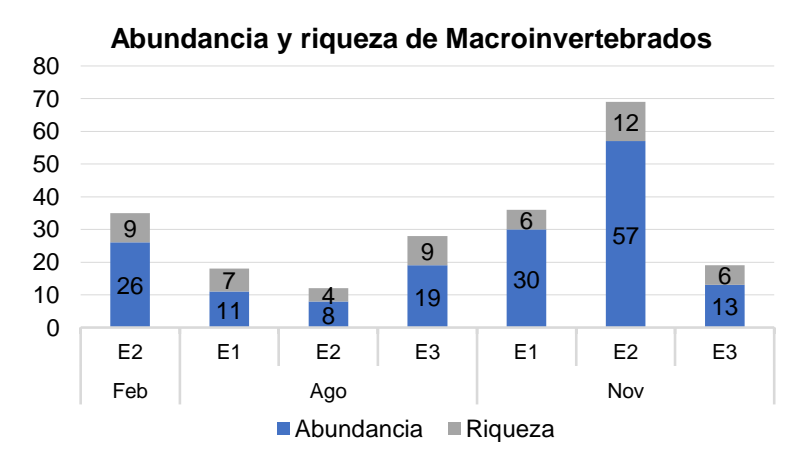

Fig. 5. Abundancia y riqueza de macroinvertebrados en las campañas de muestreo.

Tabla 7. Riqueza, abundancia y densidad de las muestras cuantitativas de macro-invertebrados

\begin{tabular}{cccccccc}
\hline \multirow{2}{*}{ Variable } & Feb. & \multicolumn{3}{c}{ Ago. } & \multicolumn{3}{c}{ Nov. } \\
\cline { 2 - 8 } & E2 & E1 & E2 & E3 & E1 & E2 & E3 \\
\hline Abundan- & 26 & 11 & 8 & 19 & 30 & 57 & 13 \\
cia & 9 & 7 & 4 & 9 & 6 & 12 & 6 \\
$\begin{array}{c}\text { Riqueza } \\
\text { Densidad }\end{array}$ & 144,4 & 61,1 & 44,4 & 105,6 & 166,7 & 316,7 & 72,2 \\
$\left(\# I^{\prime n d} / \mathrm{cm}^{2}\right)$ & & & & & & &
\end{tabular}

En cuanto a la abundancia relativa (Fig. 6.), Anacroneuria sp. se encontró en la mayoría de las estaciones, aunque no fue tan abundante, pero si siendo de mucha importancia por sus características como bioindicador. Phylloicus sp. fue hallado en mayor proporción en casi todas las estaciones durante las tres campañas de muestreo, pertenece a la familia Calamoceratidae, sus larvas pueden vivir en ambientes lénticos y lóticos, con aguas bien oxigenadas; siempre se asocia a hojarasca sumergida, de la cual se alimenta en descomposición, y pueden llegar a ser bastante abundante localmente, especialmente en las orillas de ríos y quebradas, de áreas boscosas; están ampliamente distribuidos en Latinoamérica, llegando hasta el sur de los Estados Unidos. La gran mayoría de las especies son sensibles a la contaminación del agua [27]. Según Roldán (2016) es uno de los géneros de la orden Trichóptera más sensibles a la degradación del hábitat y al aumento de carga orgánica y residuos; vive en aguas de corrientes frías [16]. 

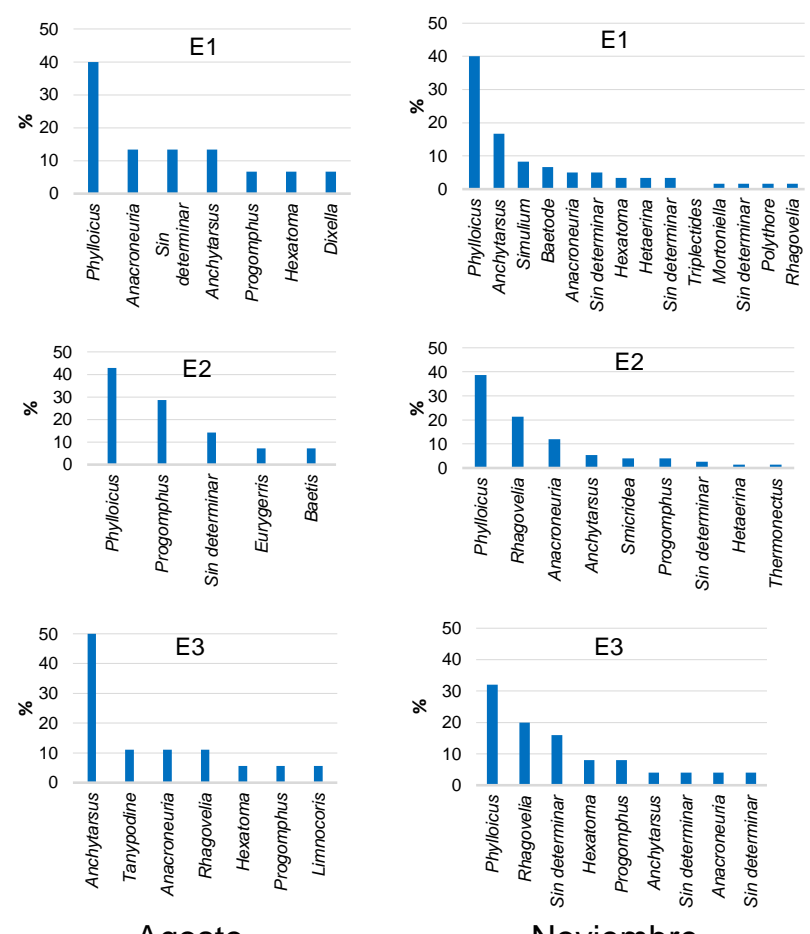

Agosto

Noviembre

Fig. 6. Abundancia relativa de macroinvertebrados acuáticos más representativos en la quebrada Santa Mónica por estación (Fuente: Elaboración propia).

Para el cálculo de los índices BMWP y \% ETP se tuvieron en cuenta tanto las muestras cualitativas como cuantitativas. La calificación del BMWP en las dos primeras campañas de muestreo, no presentaron variaciones significativas alcanzando valores entre 37 y 53 (Tabla 8.), lo que las sitúa como aguas contaminadas naturalmente, y aguas impactadas por la descomposición de materia orgánica, pero en la última campaña se hallaron aguas muy limpias, recibiendo una calificación entre 70 y 100 [1].

Tabla 8. Índices bióticos \% ETP y BMWP/Col en las diferentes campañas

\begin{tabular}{cccccccc}
\hline & FEB & \multicolumn{3}{c}{ AGO } & \multicolumn{3}{c}{ NOV } \\
\cline { 2 - 8 } ÍNDICES Y CALIDAD & E2 & E1 & E2 & E3 & E1 & E2 & E3 \\
\hline BMWP/Col & 53 & 52 & 37 & 50 & 100 & 119 & 70 \\
Calidad & Dudosa & Dudosa & Dudosa & Dudosa & Buena & Buena & Aceptable \\
$\%$ \%TP & 83,8 & 53 & 50 & 10,5 & 55 & 56 & 25 \\
Calidad & Buena & Buena & Buena & Dudosa & Buena & Buena & Aceptable \\
\hline
\end{tabular}

Los resultados para este índice revelan agua de calidad buena en todas las estaciones, excepto en E3 en el mes de agosto.

En todos los meses de muestreo, el índice de Shannon-Weaver para todas las estaciones estuvo por debajo de 3 y por encima de 1, con un promedio general de 1,31, lo que corresponde a aguas moderadamente contaminadas [28], excepto para el mes de agosto, en E2, y en el mes de noviembre, en E3, donde se dieron valores mínimos. No se presentaron variaciones significativas entre las estaciones para las diferentes campañas. Al igual que la riqueza, la dominancia de Simpson presentó algunas variaciones entre cada una de las estaciones, excepto en el mes de noviembre en que fueron similares para E1 y E2 (ver Tabla 9)

Tabla 9. Índice de diversidad y sus componentes en las diferentes estaciones.

FECHA ÍNDICES Y ESTACIONES




\begin{tabular}{cccccccccccccc}
\hline & \multicolumn{3}{c}{ Diversidad } & \multicolumn{4}{c}{ Dominancia } & \multicolumn{3}{c}{ Riqueza } & \multicolumn{3}{c}{ Equidad } \\
\cline { 2 - 14 } & & E1 & E2 & E3 & E1 & E2 & E3 & E1 & E2 & E3 & E1 & E2 & E3 \\
\hline \multirow{2}{*}{ Feb17 } & $\mathbf{R} 1$ & - & 1,61 & - & - & 0,28 & - & - & 5 & - & - & 0,6 & - \\
& $\mathbf{R} 2$ & - & 1,33 & - & 0,0 & 0,089 & - & - & 7 & - & - & 0,57 & - \\
\multirow{2}{*}{ Ag017 } & $\mathbf{R} 2$ & 1,61 & 0,60 & 1,83 & 0,00 & 0,52 & 0,18 & 5 & 2 & 9 & 0,69 & 0,60 & 0,58 \\
& $\mathbf{R 1}$ & 1,39 & 1,62 & 1,10 & 0,29 & 0,28 & 1,00 & 6 & 9 & 3 & 0,54 & 0,51 & 0,69 \\
\multirow{2}{*}{ Nov17 } & $\mathbf{R 2}$ & - & 1,41 & 0,80 & 1,00 & 0,30 & 0,56 & 1 & 6 & 3 & - & 0,57 & 0,51 \\
\hline
\end{tabular}

Los resultados del índice de Jacard (Tabla 10.) muestran que para el mes de agosto hay una similitud del $50 \%$ entre E1 y E2, igual que para E1 y E3; en el mes de noviembre se presentó una mayor similitud entre E1 y E3 situándose en el $57 \%$.

Tabla 10. Índices de similitud entre las estaciones muestreadas

\begin{tabular}{lllllll}
\hline \multirow{3}{*}{ Índices } & \multicolumn{4}{c}{ Periodos de muestreo y estaciones } \\
\cline { 2 - 7 } & \multicolumn{3}{c}{ Agosto } & \multicolumn{4}{c}{ Noviembre } \\
\cline { 2 - 7 } & $\mathrm{E} 1-\mathrm{E} 2$ E1-E3 & $\mathrm{E} 2-\mathrm{E} 3$ & $\mathrm{E} 1-\mathrm{E} 2$ & $\mathrm{E} 1-\mathrm{E} 3$ & $\mathrm{E} 2-\mathrm{E} 3$ \\
\hline Jacard (1908) & 0,50 & 0,50 & 0,17 & 0,29 & 0,57 & 0,29 \\
Sörensen (1948) & 0,67 & 0,67 & 0,29 & 0,44 & 0,73 & 0,44 \\
Coef. de similitud & 0,75 & 0,75 & 0,38 & 0,55 & 0,80 & 0,55 \\
\hline
\end{tabular}

4.

\section{6. Perfil topográfico}

Se realizó el perfil topográfico del cauce y la distribución espacial de macroinvertebrados acuáticos (Fig. 7.). Se observó que el cauce en este tramo está bordeado por taludes de alta pendiente que se aligera un poco en la estación 3. Esto se traduce en aportes por escorrentía y arrastre de material vegetal durante las lluvias, sumado al que ya de por si transporta la corriente desde aguas arriba.

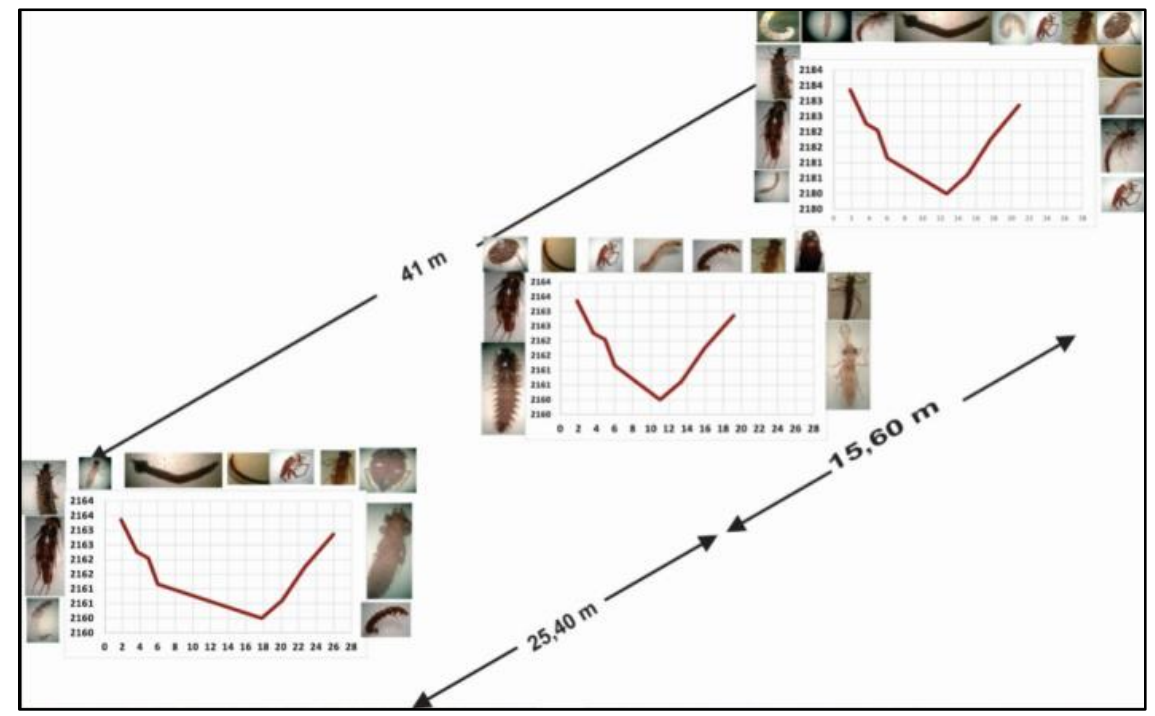

Fig. 7. Perfil topográfico y distribución espacial de macroinvertebrados acuáticos en la Quebrada Santa Mónica. (Fuente: Elaboración propia)

\section{CONCLUSIONES}

El mes de noviembre presentó el valor más alto de caudal, debido a las altas precipitaciones registradas en este periodo. En el mes de agosto se registró un caudal muy pequeño de $3,4 \mathrm{~L} / \mathrm{s}$ en $\mathrm{E} 3$, lo que indica que se 
puede afectar la disponibilidad de agua que puede llegar al embalse para el abastecimiento al municipio de Concordia en algunos periodos del año.

Según a los resultados de los datos fisicoquímicos y microbiológicos en los períodos de muestreo estudiado, se tiene que los valores de los parámetros oxígeno disuelto, $\mathrm{pH}$, conductividad, nitritos, nitratos, alcalinidad y dureza se encuentran dentro de lo establecido por la normatividad. Mientras que la DBO, turbiedad y coliformes totales y fecales no cumplen. Por lo que es necesario realizar el respectivo tratamiento especificado por la norma RAS 2017.

De las variables fisicoquímicas presentes en el mes de noviembre, las que reportaron una mayor concentración fueron oxígeno disuelto, porcentaje de saturación de oxígeno, $\mathrm{CO}_{2}$, y turbiedad, debido al aumento de la precipitación y por tanto del caudal, en época de lluvias, favoreciendo el arrastre de hojarasca desde aguas arriba; ello implica una mayor cantidad de detritus en descomposición, lo que aumenta la concentración de $\mathrm{CO}_{2}$ que a su vez influye en la abundancia de macroinvertebrados acuáticos. La alta concentración de oxígeno en todos los periodos y estaciones permitió que géneros como Phylloicus sp. y Anacroneuria sp., predominaran, ya que existen condiciones favorables para su desarrollo.

La distribución espacial y el comportamiento de la comunidad de macroinvertebrados acuáticos, su riqueza, abundancia, densidad y los resultados de los índices bióticos, permiten concluir que la quebrada Santa Mónica presenta aguas de buena calidad, con una contaminación leve. El período en que se encontró una mayor abundancia de macroinvertebrados fue en el mes de noviembre; la estación donde se presentó mayor abundancia fue la E2, asociado a un sustrato arenoso y presencia de hojarasca en el cauce.

De acuerdo con la información recolectada para definir el perfil topográfico, el cauce está bordeado por taludes de alta pendiente que se van aligerando un poco en la E3. Esto significa aportes por escorrentía y arrastre de material vegetal durante la lluvia, sumado al que ya de por sí trae la corriente desde aguas arriba.

Las aguas de la quebrada Santa Mónica, son de buena calidad y aptas como fuente de captación para el abastecimiento de agua para el consumo, aplicando los procesos de tratamiento previos recomendados por el RAS 2017. Se recomienda hacer estudios hidrológicos más amplios para conocer la disponibilidad de agua en todos los periodos del año.

\section{AGRADECIMIENTOS}

Los autores agradecen al grupo GeoLimna de la Escuela Ambiental-Facultad de Ingeniería de la Universidad de Antioquia por el apoyo en este trabajo de investigación que se realizó en el marco del proyecto CODI: Caracterización de la biodiversidad en el área de influencia de la reserva natural La Nitrera, Concordia Antioquia, con el apoyo de la Secretaría de Agricultura y las Empresas Públicas Municipales de Concordia. Al Laboratorio de Estudios Ambientales de la Universidad de Antioquia, a los estudiantes de pregrado Alejandro Espinal Restrepo y Shirley Viviana Giraldo Duque, y a la estudiante de posgrado Luisa Fernanda Pérez, quienes apoyaron los muestreos, y a; a Maikol Daniel Córdoba, técnico del Laboratorio de Hidrobiología Sanitaria-Facultad de Ingeniería de la Universidad de Antioquia.

\section{REFERENCIAS BIBLIOGRÁFICAS}

[1] N. Aguirre and O. Caicedo, "Métodos de Campo y laboratorio para Hidrobiología Sanitaria," Reimpr. Univ. Antioquia, 2013.

[2] W. Milán, O. Caicedo, and N. Aguirre, "Quebrada La Popala un análisis de calidad del agua desde algunas variables fisicoquímicas, microbiológicas y los macroinvertebrados acuáticos," Gestión y Ambient., vol. 14, no. $1,2011$.

[3] N. j. Aguirre, J. Palacio, and Á. Will, Caracterización de los principales aspectos fisicobióticos de la microcuenca de la quebrada La Vega, municipio de San Roque, Antioquia. Medellin: Grupo de Investigacion en Gestion y Modelacion Ambiental, 2004.

[4] C. N. Sawyer, P. L. McCarty, and G. F. Parkin, Química para ingeniería ambiental. McGraw Hill, 2001.

[5] A. Guerra, R. Néstor, J. Aguirre, R. Orlando, and C. Quintero, "Mapificación y analisis de la distribución espacial de organismos indicadores de la calidad del agua en la quebrada la Ayurá Mapping and spatial 
analysis of the distribution of indicator organisms for water quality in the stream of Ayura ( Envigado , Anti," vol. 14, no. 2011, pp. 1-11, 2020.

[6] E. Domínguez, M. Hubbard, M. Pescador, and C. Molineri, "Guía para la determinación de los artrópodos bentónicos sudamericanos," Macroinvertebrados bentónicos Sudam., pp. 55-631, 2001.

[7] W. Pino et al., "Diversidad de macroinvertebrados y evaluación de la calidad del agua de la quebrada La Bendición, municipio de Quibdó(Chocó - Colombia) Diversity of Macroinvertebrados and Evaluation of the Quality of the Water of the Gulch the Bendición, Municipality of," Acta Biológica Colomb., vol. 8, no. 2, 2003. [8] G. Roldán-Pérez, "Los macroinvertebrados como bioindicadores de la calidad del agua: cuatro décadas de desarrollo en Colombia y Latinoamerica," Rev. la Acad. Colomb. Ciencias Exactas, Físicas y Nat., vol. 40, no. 155, p. 254, Jul. 2016.

[9] J. A. Posada G., G. Roldán P., and J. J. Ramírez R., "Caracterización fisicoquímica y biológica de la calidad de aguas de la cuenca de la quebrada Piedras Blancas, Antioquia, Colombia," Rev. Biol. Trop., vol. 48, no. 1, 2000.

[10] B. Atehortúa Trujillo et al., "Medición y análisis de la diversidad de comunidades hidrobiológicas: una perspectiva desde la ingeniería ambiental," Rev. Politécnica, vol. 15, no. 28, 2019.

[11] Y. Montoya Moreno and A. F. Escobar Gutiérrez, "Los macroinvertebrados acuáticos y la calidad biológica del agua en una quebrada andina, Antioquia-Colombia," Rev. Politécnica, vol. 15, no. 29, 2019.

[12] m. d. C. Concejo, "Plan de Desarrollo Concordia 2016-2019." Alcaldía de Concordia, 2016.

[13] C. y T. Ministerio de Vivienda, Reglamento Técnico del Sector de Agua Potable y Saneamiento Básico - RAS 2017. 2017.

[14] N. Aguirre et al., "Caracterización de la fauna en el área de influencia de la reserva natural La Nitrera, Concordia, Antioquia, Colombia." Sin Publicar, 2020.

[15] Corantioquia, Manual piragüero. Corantioquia, EPM, 2014.

[16] G. Roldán, "Guía para el estudio de los macroinvertebrados acuáticos del departamento de Antioquia.," 1988.

[17] H. Fernández and E. Domínguez, "Guía para la determinación de los arthrópodos bentónicos Sudamericanos. Entomotropica," vol. 16(3), p. 219, 2001.

[18] G. Roldán, "Bioindicación de la calidad del agua en Colombia (Uso del método BMWP/Col). Universidad de Antioquia.," p. 170, 2003.

[19] K. R. Chesters, "Biological monitoring working part. The 1978 national testing exercise," Water Data Unit - Tech. Memo., vol. no. 19, 1980.

[20] J. Posada and G. Roldan, "Clave ilustrada y diversidad de las larvas de Trichoptera en el NorOccidente de Colombia," Caldasia, vol. 25, no. 1, pp. 169-192, 2003.

[21] Standard Methods, "Benthic Macroinvertebrates," no. 10500, 2001.

[22] W. P. McCafferty, Aquatic Entomology. Jones and Bartlett, 1983.

[23] S. Álvarez, L. Pérez, J. Mora, L. López, and E. Tenorio, Evaluación de la calidad de agua mediante la utilización de macroinvertebrados acuáticos en la subcuenca del Yeguare, Honduras. Zamorano: Escuela Agrícola Panamericana-2012, 2007.

[24] M. A. Barrionuevo, F. Romero, M. G. Navarro, G. S. Meoni, and H. R. Fernandez, "Monitoreo de la calidad del agua en un río subtropical de montaña: el río Lules (Tucumán, Argentina).," Conagua, 2007.

[25] Ideam, "Solicitud de Informacion Hidrometeorológica," 2018. [Online]. Available: http://www.ideam.gov.co/solicitud-de-informacion. [Accessed: 12-Feb-2018].

[26] J. Naranjo and Y. Montoya, "Efectos asociados al cambio del cauce del río San Lorenzo, El Carmen de Viboral-Antioquia," Rev. Politécnica, vol. 16, no. 3, pp. 112-128, 2020.

[27] M. Springer, "Capítulo 7. Trichoptera," Rev. Biol. Trop., vol. 58, no. SUPPL. 4, pp. 151-198, 2010.

[28] J. F. Wilhm and T. C. Dorris, Biological parameters of water quality. Bioscience, 1968. 